\title{
Turismo residencial y migración de jubilados extranjeros en México: un estudio de caso sobre sus implicaciones ambientales y de servicios en Baja California Sur \\ Diego Armando Casas-Beltrán, ${ }^{*}$ Luis Felipe Beltrán-Morales, ** Aradit Castellanos $^{* * *}$ y Aurora Breceda Solís-Cámara ${ }^{* * *}$
}

Resumen

La migración de jubilados extranjeros en México ha aumentado, especialmente en Los Cabos, donde los asentamientos tipo tráiler se incrementan y se desplazan a zonas rurales costeras de alto valor ecológico, con el desconocimiento de las implicaciones ambientales. El objetivo de este trabajo es contribuir al conocimiento de este tipo de asentamientos, documentando mediante un caso de estudio las características de su comunidad así como sus implicaciones ambientales y de dotación de servicios públicos. Concluimos que la localidad se convertirá en un sitio de retiro en corto plazo, pues si bien no presenta altas densidades, sus implicaciones ambientales son significativas y de una cobertura amplia. Su demanda actual de servicios es mínima, pero puede producir conflictos a futuro, especialmente con el agua.

Palabras clave: turismo residencial, migración, jubilados, implicaciones ambientales, Los Cabos.

\begin{abstract}
Migration of foreign retirees in Mexico has increased, especially in Los Cabos, where settlements trailer type are increasing and are moving into rural coastal areas of high ecological value, without the knowledge of impacts that may result. Thus the aim of this paper is to contribute to the understanding of this type of settlement, by documenting a case study, the characteristics of their community, their environmental impacts and implications for the provision of public services. We conclude that the town will become a short-term retreat site, because while it does not have high densities, its environmental implications are significant and broad coverage. Its current demand for services is minimal, but may cause conflicts in the future, especially with the water. Keywords: residential tourism, migration, retirees, environmental implications, Los Cabos
\end{abstract}

* Estudiante del Programa de Doctorado en Ciencias en el Uso, Manejo y Preservación de los Recursos Naturales del Centro de Investigaciones Biológicas del Noroeste. Correo electrónico: dcasas@cibnor.mx

** Investigador titular C del Centro de Investigaciones Biológicas del Noroeste. Correo electrónico: lbeltran04@cibnor.mx

*** Investigador titular A del Centro de Investigaciones Biológicas del Noroeste. Correo electrónico: arcas04@cibnor.mx

**** Investigadora titular A del Centro de Investigaciones Biológicas del Noroeste. Correo electrónico: abreceda@cibnor.mx 


\section{Introducción ${ }^{1}$}

El turismo se define "como todas las actividades que realizan las personas al viajar y al estar en lugares fuera de su lugar de origen, por no más de un año consecutivo" (OMT, 2005). Esta actividad es muy diversa, por lo que hay distintas formas de abordar su estudio. Cohen (1974) sugiere una clasificación de turistas (los institucionalizados, que planean sus vacaciones con agencias, y los no institucionalizados, quienes no lo hacen) basándose en la afirmación de que las experiencias de los turistas combinan varios grados de novedad relacionados a lo familiar y la excitación del cambio relacionada con la seguridad de la cotidianidad.

Smith (1977) caracteriza al turismo por el tipo de actividad preferencial de los turistas en el destino; por ejemplo, el turismo recreacional centra las actividades en el buen clima, la playa y los parajes de "interés". El turismo cultural incluye lo pintoresco, el colorido local y el folclor de las comunidades rurales. El turismo histórico hace una revisión de las glorias del pasado, representadas en museos, catedrales, monumentos y ruinas. Finalmente, el turismo medioambiental utiliza espacios naturales, como parques, y su principal atractivo es el conocimiento de la flora y la fauna de los sitios receptores.

Dentro de esta gama de tipos de turismo, se encuentra el turismo residencial, el cual Raya-Mellado (2001) define como "tipología de turistas que utilizan, con mayor frecuencia, alojamientos no reglados y cuyas estancias son relativamente mayores que las del turismo vacacional o recreacional". En este sentido, Raya denomina "alojamientos no reglados" a aquellos alojamientos no hoteleros, como viviendas propias, rentadas o prestadas, o de amigos; en este sentido, la definición de Hiernaux-Nicolás (2005) es más específica pues considera al turismo residencial como "aquel por el cual las personas acuden a un destino o una localidad que no es forzosamente turística per se, donde tienen la posesión por compra, renta o préstamo de un inmueble en el cual pernoctan y realizan actividades de ocio y esparcimiento" (Hiernaux-Nicolás, 2005).

${ }^{1}$ Se agradece a J. Rivera, G. E. Ceseña, R. Coria y P. González, personal del sig del CIBNOR, su apoyo en la elaboración de la cartografía digital. Al proyecto "Implicaciones ecológicas del desarrollo turístico-residencial en una zona costera (Los Frailes-Punta Gorda) en Baja California Sur", clave 119336. 
Así, en los últimos años el flujo de turistas residenciales estadunidenses ha aumentado aceleradamente hacia América Latina, en especial México. Estos turistas se caracterizan por ser en su mayoría jubilados con alto poder adquisitivo, lo que les permite vivir por largas temporadas fuera de casa, con importantes impactos socioeconómicos en las localidades de destino, dándose un reordenamiento socio-espacial del espacio urbano (Lizárraga-Morales, 2009). Actualmente este flujo turístico y migratorio ha tomado mayor relevancia en las localidades receptoras por la jubilación masiva de la generación babyboomer (personas nacidas durante la explosión de natalidad posterior a la Segunda Guerra Mundial, entre 1946 y principios de la década de los años de 1960) (Kiy y McEnanie, 2010). Mundialmente representan más de $60 \%$ de la población actual, con 6,700 millones de personas (UNFPA, 2008) —en el caso de los estadunidenses, se estima que son 76 millones (Dailey, 2005).

Según cálculos para México, entre 1996 y 2006 la población inmigrante estadunidense que vivía en el país bajo alguna forma migratoria aumentó de 200000 a un millón (Lizárraga-Morales, 2009). Sin embargo, al no existir un consenso en los registros de las autoridades mexicanas y estadunidenses, se desconoce el número exacto de estos ciudadanos; esto debido a que la mayoría de ellos entran al país como turistas, aun cuando viven por largas temporadas en los destinos (Lizárraga-Morales, 2009).

Hasta la fecha, el fenómeno ha recibido poco interés en la literatura académica y su investigación ha sido limitada en su alcance: ésta proviene principalmente de disciplinas como la gerontología, la geografía del turismo, la salud pública, las políticas ambientales y de los estudios sobre migración. Lo anterior con énfasis en la investigación futura, por el posible impacto que se espera se produzca entre las personas y las localidades receptoras a causa del crecimiento exponencial de jubilados estadunidenses y canadienses en América Latina (Schafran y Monkkonen, 2011).

Con el fin de abordar de manera directa los impactos en los destinos, Schafran y Monkkonen (2011) proponen una tipología de las localidades receptoras y sus asentamientos, implantando como marco de referencia los impactos a escala urbana (tipo de ciudad) y suburbana (tipo de asentamiento). Se consideran aspectos como la antigüedad de la vivienda, el grado de integración de ésta para los residentes locales, su ubicación en 
el tejido urbano y su significado histórico o social, estableciendo cuatro tipos de asentamientos:

- El centro: son comunidades de jubilados formándose en las ciudades más antiguas de México, tanto en costa como en tierra adentro (como San Miguel de Allende, Mazatlán, Mérida, Puerto Vallarta, Chapala y Ajijic). Son de tipo urbano, los migrantes ocupan y/o compran los edificios más antiguos del centro de la ciudad. Se integran más fácil a la comunidad y a sus vecinos mexicanos, aunque pueden ejercer mucha presión sobre los mercados de bienes raíces y el territorio.

- La periferia: son comunidades cerradas (privadas), menos integradas física y socialmente que las comunidades originales (aunque a veces se integran poblaciones de mexicanos de clase media), empero aún dentro del tramado urbano, y dependen de los servicios locales. Se ubican en sitios como Chapala y Ajijic, San Miguel de Allende, Puerto Vallarta, Playas de Rosarito y Ensenada. Estos asentamientos nacieron de una comunidad colonizadora, teniendo conflictos con ellos, y son propensos a vivir al margen de mexicanos y connacionales.

- El compuesto: integrado por mega-resorts residenciales, son un híbrido entre un espacio turístico exclusivo y una comunidad residencial. Son de escala masiva, con miles de unidades tendientes al aislamiento y con residentes que cubren la mayor parte de sus necesidades básicas de los servicios locales. Se sitúan en zonas rurales o aisladas, en particular cerca de pueblos costeros pequeños, donde la integración a las comunidades mexicanas es prácticamente nula, como en Loreto Bay y las cercanías de Los Cabos y La Paz.

- El tráiler: son aglomeraciones semipermanentes de casas rodantes, remolques y viviendas pequeñas, en zonas rurales aisladas presentes a lo largo de la costa (y algunas adyacentes a comunidades de playa) del Pacífico de Baja California (Playas de Rosarito y Bahía de Los Ángeles), Baja California Sur y Nayarit (Sa-Yulita y Bucerías). Al igual que las otras tipologías, son una mezcla de residentes y visitantes durante las temporadas invernales procedentes de Estados Unidos y Canadá (Coates et al., 2002). Sus residentes son en su mayoría de clase trabajadora y media, con una fuerte relación en términos de servicios y suministros (Coates et al., 2002). 


\section{Situación en Los Cabos}

Una primera asunción a tomar en cuenta es que las fronteras no tienen que ser estrictamente geográficas para producir fenómenos de tipo frontera (Newman, 2006). Baja California Sur es el segundo estado con mayor captación de turistas extranjeros residenciales en México, especialmente el municipio de Los Cabos (Lizárraga-Morales, 2008; Kiy y McEnany, 2010). Si bien Los Cabos está lejos de la frontera, de acuerdo con Gámez y Ángeles (2010), la confluencia de la inversión extranjera anclada al turismo y la migración (aunque temporales) tiene efectos importantes tanto en las comunidades locales como en los visitantes, apoyando esto a una comprensión más flexible de las fronteras que va más allá de las fronteras geográficas (Brunet-Jailly, 2005).

La tendencia actual de desarrollo en Los Cabos es la construcción y venta de viviendas y condominios para extranjeros, presentándose, de acuerdo con la tipología de Schafran y Monkkonen (2011), en dos grandes ramas: la de asentamientos compuestos — megadesarrollos residenciales en las costas (Guido, 2007) — y la de asentamientos tipo tráiler, estos últimos constituidos por viviendas de tamaño variable que se extienden más allá de los centros urbanos, en zonas costeras ecológicamente sensibles como las dunas (Lizárraga-Morales, 2010). Estos asentamientos se clasifican como "ex urbanos" porque aunque están cerca de los pueblos en realidad no son parte de ellos y a menudo, sin todas las instalaciones, carecen de referencias locales y se definen como espacios colonizados que surgen en la misma forma en distintos sitios (Huber, 2005).

Este tipo de asentamientos es una alternativa viable para personas de clase trabajadora y media provenientes de Estados Unidos y Canadá que planean jubilarse en el corto o mediano plazos, misma que puede acentuarse por la recesión en Estados Unidos y la pérdida del poder adquisitivo. Sin embargo, esta realidad, conjugada con la falta de regulación de las autoridades locales sobre la gestión del uso de suelo, torna preocupante a las zonas costeras rurales ya que tarde o temprano estos nuevos asentamientos demandarán más infraestructura y servicios (especialmente agua, recurso escaso en la entidad), elevando los gastos corrientes y de inversión, rara vez compensados por los ingresos que generan estos turistas (Bernier, 2003), además de las implicaciones ambientales sobre sitios sensibles como las dunas. 
El objetivo del presente trabajo es contribuir al conocimiento de las características e implicaciones ambientales y a la dotación de servicios públicos que generan los asentamientos turísticos residenciales tipo tráiler, de jubilados extranjeros, mediante un caso de estudio en una zona rural costera.

\section{Materiales y métodos}

\section{Área de estudio}

El área de estudio se ubica al sur del estado de Baja California Sur, entre las coordenadas $24^{\circ} 00^{\prime} \mathrm{N}, 109^{\circ} 36^{\prime} \mathrm{O}$ a $23^{\circ} 50^{\prime} \mathrm{N}, 109^{\circ} 32^{\prime} 50^{\prime \prime} \mathrm{O}$ (figura 1). La fisiografía se compone por lomeríos bajos y planicies bajas (menores a los $100 \mathrm{~m}$ ) de material granodirítico, que desembocan en playas arenosas, con cordones de dunas semiestabilizadas intercaladas (Pérez-Navarro, 1995). Dos comunidades vegetales integran la vegetación: el Matorral Sarcocaule (MS) y la Vegetación de Dunas Costeras (vdc) que forman parte de la región florística del Cabo de la península de Baja California, reconocidas como hábitats de interés para conservación (Pérez-Navarro, 1995; León de la Luz, 1999).

El ms es una variante del matorral xérofilo (Rzedowski, 2006), con dominancia de elementos arbustivos y compuesta por cerca de 400 especies, 10\% endémicas (León de la Luz, 1999). La vDC es una comunidad de baja altura, compuesta por hierbas y arbustos, con especies endémicas del ambiente costero de la Región del Cabo (Pérez-Navarro, 1995). Las playas arenosas (PA) son franjas de material arenoso claro, de hasta 400 metros en sentido perpendicular a la costa, hasta el primer cordón de dunas semiestabilizadas (Pérez-Navarro, 1995). Para cumplir los objetivos, el trabajo se realizó en cuatro etapas y tres periodos: 1978-1999, 1999-2007 y 19782007 (figura 1). 
Figura 1. Área de estudio

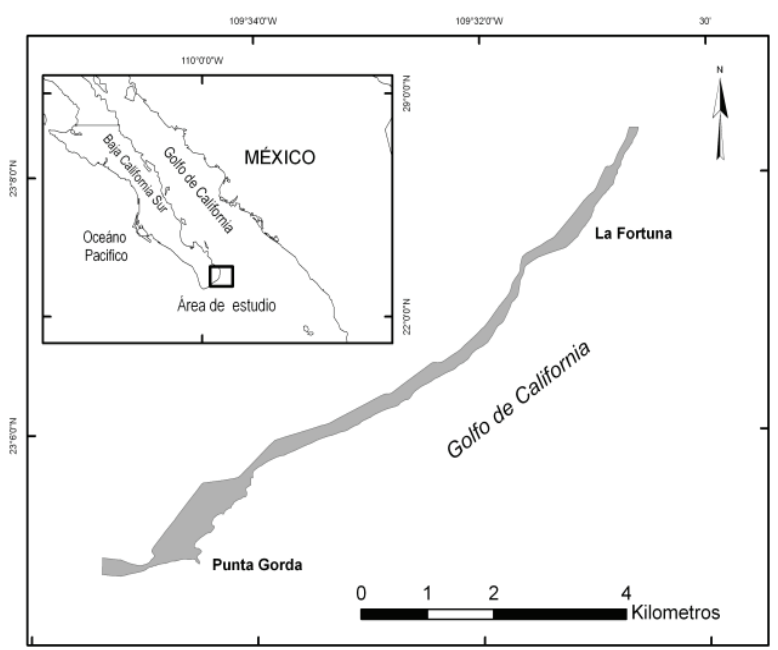

Fuente: Elaboración propia.

\section{Metodología}

Tipificación y delimitación de tipos de vegetación, hábitat y usos de suelo

Los tipos de vegetación identificados (MS y VDC) corresponden a los definidos por León de La Luz (1999) y Pérez-Navarro (1995); las categorías de uso de suelo (viviendas, caminos y áreas de desmonte) fueron tomadas de González-Abraham et al. (2007) y Hawbaker (2004); las playas arenosas se definieron como categoría de hábitat (PA). La delimitación de los tipos de vegetación, hábitat y usos de suelo (TVHus) en imágenes y cartografía, se basó en criterios o atributos físicos (coloración y tipo de suelo) y ecológicos (fisonomía de la vegetación, distribución, tamaño y forma de parches).

\section{Digitalización de TVHUS e integración de mapas}

La delimitación de los TVHus se hizo mediante un análisis visual simple, sus bordes se digitalizaron en pantalla, auxiliándose del programa ArcMap 
versión 9.2 en formato vectorial, a una escala de 1:80 000. La digitalización de los caminos de 1978 se basó en el análisis visual de las fotografías aéreas de ese año; las de 1999 y 2007 se basaron en la carta topográfica del Instituto Nacional de Estadística, Geografía e Informática, INEGi (1994a; escala 1:50 000), agregando las vialidades detectadas en el análisis visual de las fotografías aéreas correspondientes a cada año. Finalmente, todas las capas vectoriales de TVHus de cada año se sobrepusieron y se integraron a un solo mapa, para cada uno de los años de estudio. Este mapa se convirtió a formato raster con el programa IDRISI TAIGA para facilitar el análisis de los cambios de TVHus.

\section{Evolución de la zona de disturbio}

Para valorar las implicaciones ambientales asociadas a las viviendas, se utilizó el indicador de Zona de Disturbio (zD) definida como el área en torno a una construcción (viviendas y caminos) en la que la calidad del hábitat se degrada de forma similar al efecto de borde en parches de vegetación (ver Theobald et al., 1997). Para simular esto, se consideró toda el área terrestre potencialmente afectada por cada edificio y camino, independientemente de su tipo de cobertura. Con base en el concepto de zD, se modeló el efecto de cada construcción, utilizando una franja de $100 \mathrm{~m}$ alrededor de las viviendas, a ambos lados de los caminos y a la combinación de ambos, a la que se sobrepuso a los mapas de cobertura y uso de suelo para cada año y se restó a las superficies de cada categoría, utilizando el programa ArcMap. La ZD de viviendas, de acuerdo con Theobald et al. (1997), se encuentra en función de dos componentes: el número de viviendas (densidad) y el patrón espacial de su ubicación (dispersión) — para los caminos, sólo está en función de la densidad, por lo cual se calcularon dichos componentes.

\section{Densidad de viviendas y de caminos e índice de dispersión de viviendas}

La densidad de viviendas y de caminos y el índice de dispersión de viviendas se emplearon como indicadores del grado de evolución de la urbani- 
zación. La densidad de viviendas se calculó como el número de viviendas por kilómetro cuadrado (número de viviendas $/ \mathrm{km}^{2}$ ), la densidad de caminos como kilómetros lineales de caminos por kilómetro cuadrado $(\mathrm{km}$ lineales de caminos/ $\mathrm{km}^{2}$ ). El índice de dispersión (Ids) se calculó estableciendo una gradilla de 300 x $300 \mathrm{~m}$ sobre los mapas de coberturas de cada año, en la que se contabilizó el número de viviendas por celda (ver González-Abraham et al., 2007). Para evaluar su dispersión se utilizó el estadístico de dispersión de Fisher et al. (1922) el cual se basa en el promedio (x) y la varianza (Var) del número de viviendas en cada celda:

$$
I d s=\operatorname{Var} / x \quad(\text { ecuación } 1)
$$

Este índice, si la razón es más de 1, indica agrupamiento; un número más alejado de 1 , indica un mayor grado de agrupamiento. Por el contrario, un número inferior a 1 indica dispersión.

\section{Aplicación de encuestas}

A fin de obtener la mayor información posible y debido a la falta de registros oficiales del número de personas extranjeras que habitan en la zona, se decidió entrevistar a los dueños de todas las viviendas (88) dentro del área de estudio. Así, se realizó una encuesta vivienda por vivienda en marzo de 2010, lográndose la participación y respuesta, por los dueños o sus representantes, en 75 viviendas, dando por resultado un $6 \%$ de error permitido a un $99 \%$ de nivel de confianza, determinado a partir de la fórmula propuesta por Suárez (2004):

$$
n=\left(N \sigma^{2} Z^{2}\right) /\left(e^{2}(N-1)+\sigma^{2} Z^{2}\right) \quad(\text { ecuación } 2)
$$

Donde $N$ es el tamaño de la población (88 viviendas), $\sigma$ la desviación estándar de la población (0.5), $Z$ es el valor obtenido mediante niveles de confianza (2.58 a $99 \%$ de nivel de confianza) y $e$ es el límite aceptable de error muestral (0.06 a un 6\% de error). La encuesta consistió en un cuestionario, aplicado mediante una entrevista semiestructurada en la que se tomaron datos básicos tanto del entrevistado como de los ocupantes de 
la vivienda y que tuvo como objetivo evaluar si las características de los turistas de la zona corresponden a la tipología de tráiler de Schafran y Monkkonen (2011), por lo cual se determinó su nacionalidad, su escolaridad (si eran de clase trabajadora o media), su estatus migratorio y tiempo de estancia (si son una mezcla de residentes y visitantes), su demanda de servicios de electricidad, drenaje, telefonía, recolección de residuos sólidos y especialmente consumo de agua por ser un recurso escaso en la entidad — consumo en temporada alta y baja, su abastecimiento (red municipal, empresas transportadoras, pozos propios o de salación)—. Además, determinar sus edades (si están en edad de retirarse) y el tipo de propiedades (propia, rentada o prestada, o si comprarían o consideran residir en un futuro, lo que convertiría al sitio en un destino de retiro).

\section{Resultados}

\section{Implicaciones ambientales: cambios de cobertura}

El cambio de cobertura produjo una disminución de $18.4 \%$ del total de la superficie de vegetación y hábitats originales en los casi 30 años del estudio. El mayor cambio se registró en el MS, seguido de la VDC, en tanto que las PA tuvieron cambios menores. Los incrementos de las coberturas de viviendas, caminos y desmonte, en ese orden de importancia, fueron los cambios dominantes. Hubo, además, cambios asociados a la dinámica natural, de procesos de sucesión o acreción de dunas y playas. Así, las viviendas, ausentes en 1978, alcanzaron en 2007 7.6\% del total de la superficie. Los caminos pasaron de $0.4 \%$ en 1978 a $7.1 \%$ del total de la superficie en 2007. El desmonte se incrementó de $0.31 \%$ en 1978 a $3.7 \%$ del total de la superficie en 2007 (cuadro 1).

\section{Densidad de viviendas y caminos}

En 1978 no había viviendas construidas. La densidad promedio en 1999 era de 11 viviendas $/ \mathrm{km}^{2}$, y se triplicó en 2007 (cuadro 2). La densidad de 
Cuadro 1. Cobertura de tipos de vegetación y uso de suelo en el área de estudio en 1978, 1999 y 2007 y tasas de cambio porcentual entre periodos

\begin{tabular}{lccc}
\hline Tipos de vegetación y uso de suelo & 1978 & 1999 & 2007 \\
\hline MS & $128(53.9)$ & $101(42.5)$ & $93(39.2)$ \\
VDC & $34(14.5)$ & $32(13.4)$ & $28(11.7)$ \\
PA & $73.3(30.9)$ & $77.6(32.8)$ & $72.7(30.7)$ \\
Viviendas & $0(0)$ & $6(2.4)$ & $18(7.6)$ \\
Caminos & $1(0.4)$ & $17(7.0)$ & $17(7.1)$ \\
Desmonte & $1(0.31)$ & $4(1.9)$ & $8.69(3.7)$ \\
Total tipos de vegetación & & & \\
\hline
\end{tabular}

Valores indican superficie en hectáreas. Los valores entre paréntesis indican porcentajes. El signo negativo indica pérdida. $\mathrm{MS}=$ Matorral Sarcocaule, $\mathrm{VDC}=$ Vegetación de Dunas Costeras, $\mathrm{PA}=$ Playas Arenosas.

Fuente: Elaboración propia.

\section{Cuadro 2. Densidades de viviendas y caminos, índice} de dispersión de viviendas y zonas de disturbio

\begin{tabular}{ccccccc}
\hline & $D C M N$ & $D C S S$ & $I d s$ & $Z_{-} C S S(\%)$ & $Z_{-} C M N(\%)$ & $Z_{-} D_{U R B}(\%)$ \\
\hline 1978 & 1.03 & 0 & $\mathrm{ND}$ & $0.00(0)$ & $21.11(9)$ & $21.11(9)$ \\
1999 & 12.54 & 11 & 6.51 & $42.65(18)$ & $171.93(73)$ & $175.02(74)$ \\
2007 & 12.57 & 29 & 4.47 & $115.01(49)$ & $172.93(75)$ & $186.82(79)$ \\
\hline
\end{tabular}

ND: Dato no disponible; DCMn: Densidad de caminos ( $\mathrm{km}$ lineales de caminos $/ \mathrm{km}^{2}$ ); DCss: Densidad de viviendas (número de viviendas $/ \mathrm{km}^{2}$ ); Ids: Índice de dispersión; zD css: Zona de disturbio por construcción de viviendas en ha (porcentaje); ZD CMN: Zona de disturbio por construcción de caminos en ha (porcentaje); y zD URB: Zona de disturbio por construcción de viviendas y caminos (porcentaje). Fuente: Elaboración propia. 
caminos en 1978 fue de $1.3 \mathrm{~km} / \mathrm{km}^{2}$, en 1999 se construyó $98 \%$ del total de caminos y su densidad fue de $16.2 \mathrm{~km} / \mathrm{km}^{2}$; en 2007 la densidad aumentó $1 \%$ (cuadro 2 ).

\section{Dispersión de viviendas}

Por la ausencia de viviendas en 1978, no se registraron datos. En 1999 surgen las primeras construcciones, agrupadas en la parte sur del área de estudio (figura 2), indicado por un índice de dispersión alto (6.51). Para 2007, se establecen nuevas viviendas cercanas a las construidas en 1999, así la agrupación disminuye (a 4.47) por nuevos grupos de viviendas en la parte norte y centro (figura 2).

Figura 2. Evolución del cambio de uso de suelo en el área de estudio

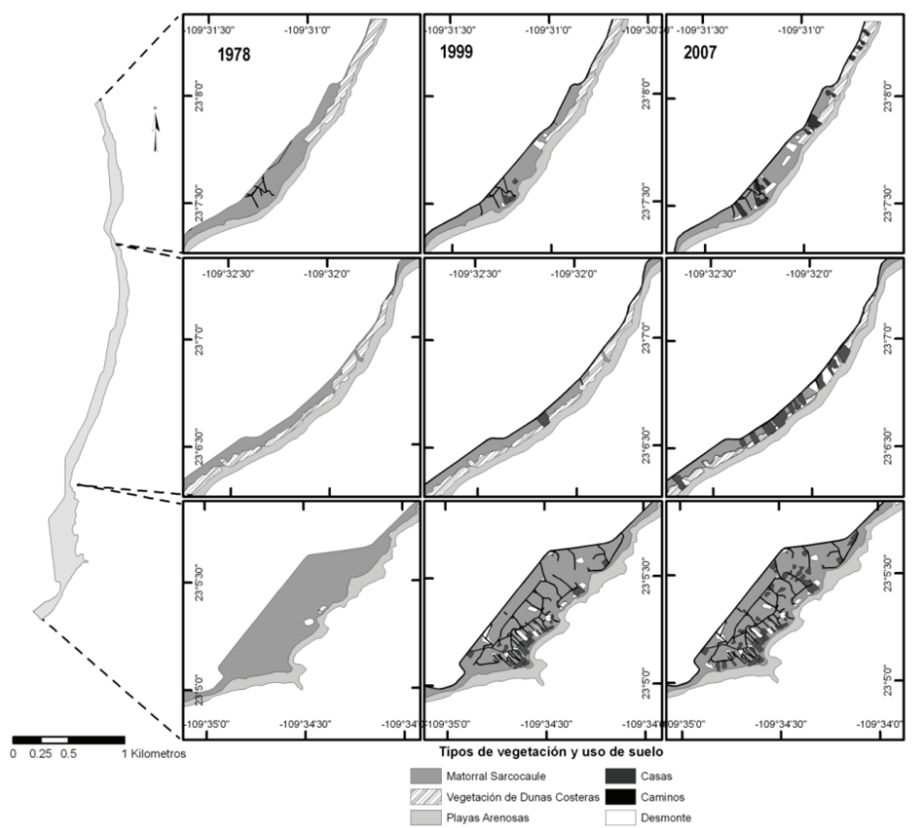

Fuente: Elaboración propia. 


\section{Zona de disturbio}

En 1978, la zD fue de 9\% del total del área de estudio, y asociado a los caminos, su apertura en 1999 produjo su amplitud (73\%). Al comenzar la construcción de viviendas la ZD fue menor a la de los caminos. En 2007, la zD de viviendas y caminos se incrementó 6\%, por la construcción de viviendas, con una apertura mínima de caminos (cuadro 2). En 1978, las ZD ocuparon $11 \%$ del MS, por la apertura de caminos, y en 1999 cubrieron 97\% del ms (cuadro 3).

En 2007, la ZD, por urbanización, se incrementó en 1\%, no obstante que la zD de viviendas aumentó a más del doble a causa de la construcción de viviendas sobre las ZD anteriormente creadas por los caminos (figura 3).

Cuadro 3. Afectación de zonas de disturbio en matorral sarcocaule

\begin{tabular}{lccc}
\hline & Viviendas & Caminos & Urbanización* $^{*}$ \\
\hline 1978 & $0(0)$ & $14(11)$ & $14(11)$ \\
1999 & $22.5(22)$ & $89.5(96)$ & $89.9(97)$ \\
2007 & $54.1(58)$ & $97.9(97)$ & $98.4(98)$ \\
\hline
\end{tabular}

Los valores indican superficie en hectáreas; los valores entre paréntesis indican porcentajes.

* Urbanización es la zona de disturbio por la construcción de viviendas y caminos.

Fuente: Elaboración propia. 
Figura 3. Evolución de la zona de disturbio de viviendas y caminos en el área de estudio

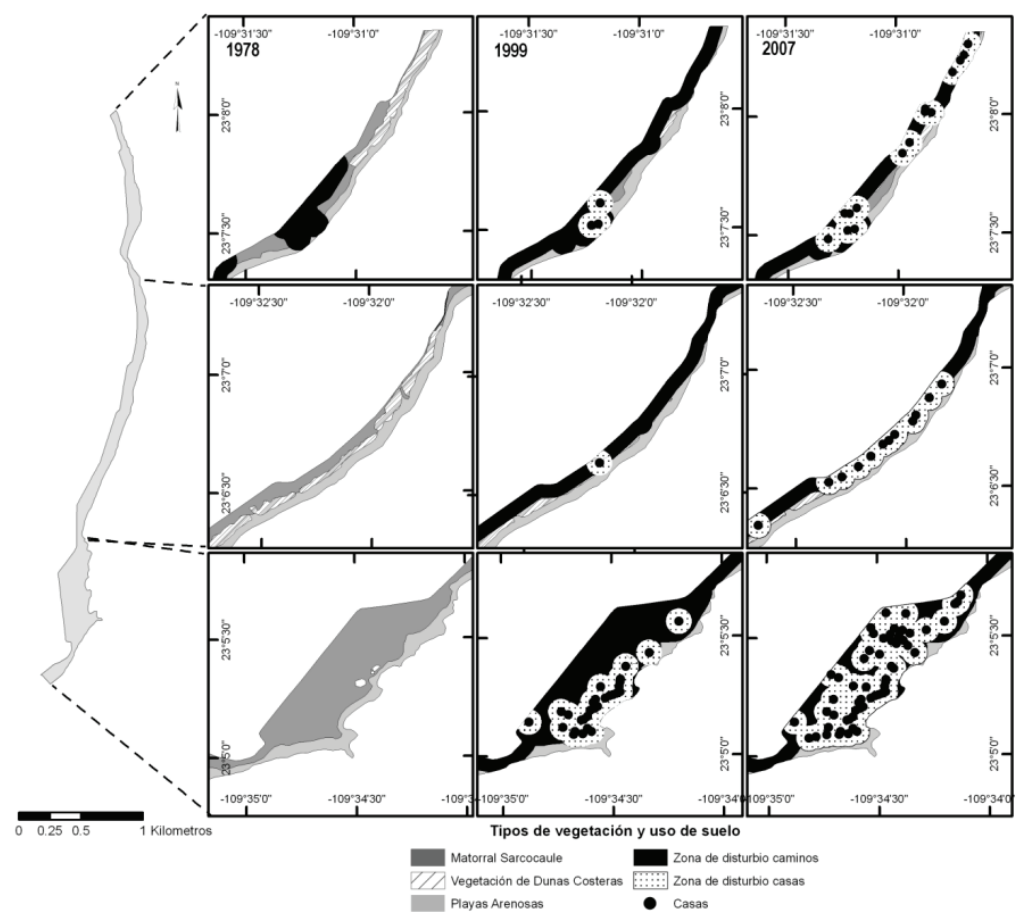

Fuente: Elaboración propia.

La VDC tuvo pérdidas similares al MS. La ZD de caminos en 1978 abarcó 5\% del total de su área; en 1999 aumentó a 69\%, principalmente por la construcción de caminos cercanos a la VDC. La zD por urbanización llegó a $76 \%$ en 2007, por las viviendas sobre las dunas (cuadro 4). En 1978 la zd de caminos ocupó 5\% de las PA, en 1999 35\% y 45\% en 2007 (cuadro 5).

Implicaciones sociales: perfil demográfico

El perfil demográfico está constituido por extranjeros —estadunidenses (83\%) y canadienses (17\%)—, donde $86 \%$ se encuentra entre los 48 y 66 años, formando parte de la generación babyboom, con una edad promedio 


\section{Cuadro 4. Afectación de zonas de disturbio en vegetación de dunas costeras}

\begin{tabular}{lccc}
\hline & Viviendas & Caminos & Urbanización* $^{*}$ \\
\hline 1978 & $0(0)$ & $2(5)$ & $2(5)$ \\
1999 & $0.4(1)$ & $21.9(69)$ & $21.9(69)$ \\
2007 & $9.4(34)$ & $19.5(71)$ & $20.9(76)$ \\
\hline
\end{tabular}

Los valores indican superficie en hectáreas; los valores entre paréntesis indican porcentajes.

* Urbanización es la zona de disturbio por la construcción de viviendas y caminos.

Fuente: Elaboración propia.

Cuadro 5. Afectación de zonas de disturbio en playas arenosas

\begin{tabular}{cccc}
\hline & Viviendas & Caminos & Urbanización \\
\hline 1978 & $0(0)$ & $4(6)$ & $4(6)$ \\
1999 & $8.3(11)$ & $24.8(32)$ & $27.4(35)$ \\
2007 & $18.1(25)$ & $24.8(34)$ & $32.6(45)$ \\
\hline
\end{tabular}

Los valores indican superficie en hectáreas; los valores entre paréntesis indican porcentajes.

* Urbanización es la zona de disturbio por la construcción de viviendas y caminos.

Fuente: Elaboración propia. 
de 56 años. El 63\% cuenta con carrera universitaria, 3\% con maestría o doctorado y el resto con al menos la educación media (college); en tanto, $57 \%$ de los encuestados está retirado, $49 \%$ se considera residente y $88 \%$ posee vivienda propia; $6 \%$ renta y el $6 \%$ restante hace uso de instalaciones prestadas. El número de meses de estancia varía de uno a 10, con un promedio de cinco, de noviembre a abril.

\section{Implicaciones sobre la dotación de servicios}

Cien por ciento de los encuestados contestó no estar conectado a la red de agua potable y alcantarillado, y proveerse de agua a través del servicio de empresas particulares de la ciudad de San José del Cabo; en cuanto a sus descargas de aguas residuales, 100\% de los encuestados afirmó que utilizaba fosa séptica. El promedio de personas que habitan las viviendas fue de dos, con un consumo de agua diario promedio anual de 158 litros/persona/día: en temporada baja 127 litros/persona/día, y 183 litros/persona/día en temporada alta. En cuanto a electricidad, servicios de telefonía e internet, 100\% de los encuestados mencionó no estar conectado a las redes de conducción y abastecerse mediante celdas solares, y comunicarse por internet satelital.

\section{Discusiones}

\section{Implicaciones ambientales}

Durante las tres décadas, se produjo una considerable pérdida de las coberturas originales (18.4\% del total) por la construcción de viviendas, caminos y el desmonte. Su magnitud supera al promedio nacional $(7.1 \%)$ de pérdida de coberturas en los dos primeros kilómetros de la línea de costa (Seingier et al., 2009) y es cercana a la observada (19.8\%) en zonas de alto desarrollo turístico residencial como Cancún, Quintana Roo (PérezVillegas y Carrascal, 2000).

La dinámica de cambio ha actuado de manera diferenciada entre los periodos, siendo mayor sobre el Ms durante el periodo 1978-1999, al cons- 
truirse los caminos y el tramado urbano, junto con las primeras viviendas, y en el periodo de 1999-2007 con la expansión de la construcción de viviendas, afectando principalmente a la VDC. Los resultados muestran que a nivel local, en los últimos años del estudio, ocurrió un proceso rápido y severo de pérdida de coberturas y degradación del terreno, con muy poca recuperación de la superficie afectada; comportamiento similar al observado en otros desarrollos en México, como Acapulco y Cancún (Carrascal y Pérez-Villegas, 1998; Pérez-Villegas y Carrascal, 2000), pero todavía distante a lo ocurrido en las costas de España, que registra $34 \%$ del primer kilómetro de línea de costa urbanizado (Guaita et al., 2006).

La dinámica de cambio de uso de suelo entre periodos puede deberse al polo de desarrollo de Los Cabos, establecido en 1974 como Centro Integralmente Planificado (Bringas-Rábago, 1999) para detonar el desarrollo económico en la región. El crecimiento acelerado de infraestructura hotelera ocurrió en un ciclo de crecimiento y auge de 25 años, y actualmente se considera en una etapa de estancamiento (Jasso-Barbosa, 2007). Esto es relevante ya que en otros destinos (como Acapulco y Cancún) se ha documentado que al llegar éstos a una etapa de estancamiento, se insertaron en una nueva fase de crecimiento: de reconversión intensiva de un modelo hotelero, hacia uno turístico residencial (Hiernaux-Nicolas, 2005). Ambas fases de crecimiento se pueden identificar en nuestra área de estudio, en donde se estableció primero el núcleo de las ciudades de Cabo San Lucas y San José del Cabo y se expandió a las periferias, comportamiento que coincide con la teoría de las causalidades acumulativas de Myrdal (Fujita, 2004), en la que nuevos centros de desarrollo crecen en la periferia de un núcleo central. En nuestro caso, se observa que en el inicio (1978) y durante el auge hotelero no existían viviendas, sin embargo, cuando empieza el estancamiento de Los Cabos (1999) comienza la reconversión hacia el modelo de segunda residencia con la construcción de viviendas, el cual se ha mantenido hasta la fecha.

En relación con las ZD, no obstante que las viviendas se encuentran agrupadas, sus ZD alcanzan 49\% del total del área de estudio en el 2007, valor elevado si consideramos que se esperaría que una forma agrupada produjera una menor zD que la dispersa (Theobald et al., 1997). Esto probablemente se debe, como lo sugieren Kiy y McEnany (2011), a que la mayoría de los propietarios de segundas viviendas son jubilados que tien- 
den a establecerse en pequeñas comunidades de amigos, a las que llegaron con recomendación de los mismos e invitando a otros para establecerse en el mismo sitio, lo cual encaja en el hecho de que se presentan pequeñas agrupaciones de viviendas aisladas en el área de estudio. Así, la pérdida de cobertura debería ser mucho menor pese al aumento en el número de viviendas (González-Abraham et al., 2007), lo que podría explicarse por los agrupamientos de viviendas, que se encuentran aislados o dispersos en la zona de estudio (principalmente en la parte centro y norte), impidiendo la sobreposición de las ZD típicas de viviendas agrupadas, pues las zonas de disturbio preexistentes se mantienen como tales, y las nuevas viviendas crean nuevas.

Los valores de pérdida de cobertura son menores que los de las zD, y son más evidentes en el Ms, donde se observa $58 \%$ de la superficie con algún grado de perturbación y una elevada pérdida (27.4\%) de la cobertura original. Esto asociado a las condiciones fisiográficas del terreno (pendientes suaves), la estabilidad del suelo (tipo granodiorítico) y a la fisonomía del ms, que permite un acceso mucho más fácil a través del mismo que en otros tipos de vegetación (Brooks y Lair, 2005), resultando económica y funcionalmente más viable la construcción de viviendas y caminos, como lo sugiere el alto número de viviendas en la parte sur del área de estudio en la que predomina el Ms.

La zD de caminos entre 1978 y 1999 tuvo un cambio de 1.03 a 12.52 $\mathrm{km} / \mathrm{km}^{2}$, y se mantuvo para el periodo de 1999 a 2007 con valores que superan a los reportados en Estados Unidos $\left(1.2 \mathrm{~km} / \mathrm{km}^{2}\right)$ y Holanda $(1.5$ $\mathrm{km} / \mathrm{km}^{2}$ ) (Forman y Alexander, 1998), y que pueden comprometer la viabilidad de ecosistemas, sobre todo en zonas áridas (Brooks y Lair, 2005). El incremento en la densidad de caminos produjo una zD que abarcó $79 \%$ del área de estudio, por la apertura del camino principal a lo largo de la línea de costa, que conecta a la localidad a la ciudad de San José del Cabo, el cual produjo una zD lineal que abarcó 97\% del Ms, vegetación en donde se abrieron casi todos los caminos; sin embargo, este disturbio fue más allá, ya que la VDC registró $1 \%$ de pérdida de su área original por apertura de caminos, pero $69 \%$ de superficie con algún grado de alteración por su cercanía a ellos. Caso similar para las PA, donde una pérdida de $6 \%$ de su superficie original representó $34 \%$ de superficie con algún grado de disturbio. 
Los resultados muestran que la pérdida de coberturas y la ZD podrían tener efectos serios en los ecosistemas locales, ya que se ha documentado que el desarrollo de la vivienda y los caminos provoca la destrucción de la vegetación natural, la alteración del suelo y la erosión, introduce especies exóticas a través de paisajes y limita los movimientos de fauna silvestre debido a los caminos y las cercas (Hostetler, 1999); además, impone patrones en el paisaje e influencia procesos ecológicos a distintas escalas (Theobald et al., 1997; Brown, 2003; Hansen et al., 2005).

Las implicaciones ambientales de la pérdida de coberturas y las ZD sobre el MS y la VDC son relevantes, al ser comunidades con alto grado de endemismo regional (León de la Luz, 1999). Así, las funciones reguladoras de la VDC ante huracanes e inundaciones podrían resultar afectadas ya que su nivel de disturbio es más del doble del promedio nacional $(7.1 \%)$ (Seingier et al., 2009) y es similar a lugares altamente perturbados, como la costa francesa, con más de $25 \%$ de la superficie original disturbada (Curr et al., 2000), lo que incidiría en su vulnerabilidad y comprometería la seguridad de las construcciones en la zona costera, como ha ocurrido en otros sitios (Pérez-Villegas y Carrascal, 2000). Lo anterior se agrava si consideramos que la recuperación de las comunidades biológicas impactadas en zonas áridas es muy lenta, comparado con zonas templadas subhúmedas o cálido-húmedas (Rosete-Vergés et al., 2008).

\section{Implicaciones sociales: perfil demográfico}

El perfil demográfico es el de un grupo babyboomer en su mayoría, con $86 \%$ entre los 48 y 66 años y un promedio de 56; el porcentaje es mayor a lo reportado (63\%) por Kiy y McEnanie (2010), lo cual puede indicar que este sitio no es atractivo para adultos mayores que requieren atención médica especializada.

La mayoría de las personas cuenta con altos niveles de educación, con carreras universitarias terminadas y estudios de maestría o doctorado (63\% y $3 \%$ respectivamente), lo que concuerda con la tipología del población de tráilers de Schafram y Monkkonen (2010) y con los datos reportados por Kiy y McEnanie (2010), en donde 60\% de los encuestados finalizó la educación superior y $28 \%$ contaba con al menos un año 
de estudios universitarios. Esto coincide con el campo de la investigación sobre la migración rural, denominado contraurbanización, siendo un concepto amplio, controvertido y discutido por mucho tiempo (Lewis et al., 1989; Vartiainen, 1989; Hoggart, 1997; Mitchell, 2004). Así, los nuevos inmigrantes a las regiones rurales a menudo no siguen las teorías económicas tradicionales de la migración. Tienen mayores ingresos, más educación y ocupaciones no tradicionales de las zonas rurales estándares, y no buscan beneficios socioeconómicos (Saint et al., 2005), sino un clima templado y las políticas favorables del gobierno, como impuestos sobre la renta y el gasto relativo al bienestar para jubilados con edades menores a los 65 años; y en el caso de personas de edad mayor, esto se reduce a factores tales como bajos costos de vida, renta e impuestos de propiedad (Conway y Houtenville, 2003).

$\mathrm{Su}$ estatus migratorio es una mezcla de residentes y visitantes en todo el año, que concuerda con la tipología de Coates et al., (2002), pero con un número alto de residentes (49\%), que indica que el sitio se está convirtiendo en un destino de retiro y se consolidaría en el corto plazo debido a que los miembros más antiguos de la generación babyboomer ya se han retirado, la mayoría se retiró entre 2005 y 2012, y los más jóvenes se retirarán en 2017 (Jauhiainen, 2009).

Así, no es raro que la gente pase su tiempo entre dos o más sitios, llegando a estar más en sus segundas residencias que en su lugar de origen (Jauhiainen, 2009); así, nuestros "visitantes" están hasta 10 meses. Vivir en dos lugares es evidente en estudios migratorios de jubilados del norte de Europa en el Mediterráneo (Casado-Díaz et al., 2004), lo que trae como resultado que la diferencia entre migración permanente y visita temporal se vuelva difusa.

El elevado porcentaje de residentes puede asociarse al hecho de que los jubilados que deciden retirarse en el extranjero visitaron la comunidad que eligieron antes de comprar una propiedad para su retiro. De hecho, la mayoría descubre estos sitios como turistas o cuando los visitan durante largos periodos de tiempo y se hospedan en una propiedad rentada o en un condominio de tiempo compartido (Lizárraga-Morales, 2009), lo cual puede estar presentándose en esta comunidad, como ha ocurrido en Cabo San Lucas, donde Lizárraga-Morales (2009) reporta que entre 95 y $97 \%$ de quienes respondieron a su encuesta habían visi- 
tado Los Cabos por lo menos una vez antes de comprar una propiedad para su retiro.

El comprar una vivienda para uso vacacional o como posible hogar de retiro, se confirma al observar que $88 \%$ de los encuestados posee vivienda propia, $6 \%$ renta y otro $6 \%$ la tiene en calidad de préstamo, resultando que el destino turístico se ha transformado en un destino de retiro, por el alto número de personas en edad de jubilarse, y que $49 \%$ ya es residente permanente.

\section{Implicaciones sobre la dotación de servicios}

El efecto de esta población sobre la dotación de servicios por el momento es muy bajo ya que no se encuentran conectados a la red eléctrica ni al sistema de agua potable y alcantarillado, y no cuentan con servicios de recolección de residuos sólidos — son los mismos propietarios los que se encargan de llevarlos al centro de acopio-. En sí, cada vivienda es autosuficiente en todos los servicios de este tipo, al contar tanto con celdas solares para generar electricidad como con aljibes —el abastecimiento de agua es mediante empresas transportadoras particulares (pipas)—; además dan tratamiento a sus propias aguas residuales mediante fosas sépticas, las cuales cuentan con pozo de absorción en el mismo terreno, lo que mitiga y minimiza el potencial peligro de contaminación de acuíferos por aguas residuales no tratadas. Por otro lado, al ser una zona rural de baja densidad poblacional y carente de vialidades pavimentadas, el peligro por infiltración a los acuíferos por superficies impermeables es muy bajo, de acuerdo con lo criterios de Beach (2002). El mantenimiento de las fosas se realiza a través de empresas particulares, en promedio una vez al año, y son éstas las encargadas de transportar y disponer de los residuos (sólidos y líquidos), reduciendo el riesgo de derrames.

El impacto que genera este tipo de asentamientos es bajo y difícil de medir. Que no estén conectados a la red de servicios públicos conlleva en sí un impacto muy bajo, al no aumentar la dotación de servicios en una comunidad alejada, sin embargo, la dotación de agua, aunque no provista por el municipio sino por particulares, sí constituye una presión a los acuíferos debido a que el líquido proviene del acuífero de San José, que presenta sobreexplotación con un déficit de $5560000 \mathrm{~m}^{3}$ al año (Cona- 
gua, 2005). De acuerdo con la Comisión Nacional del Agua (Conagua), el volumen del líquido extraído en 2007 representó en el caso del acuífero de San José del Cabo 109\% de la recarga natural anual. Este acuífero es costero, por lo tanto vulnerable a la intrusión salina. Se desconoce una regla general que permita definir el umbral de extracción que evite la intrusión salina de un acuífero. La intrusión salina depende de la configuración física del acuífero respecto del nivel del mar, por lo que no es evidente la determinación de un valor máximo deseable de explotación. En México existen tres acuíferos reportados con intrusión salina y sobreexplotación publicados en el Diario Oficial, uno en Colima, con una explotación de $68 \%$ del volumen que recarga naturalmente al año, y otros dos en Veracruz -acuífero Costera de Coatzacoalcos y Costera de Veracruz-, con una explotación de $21 \%$ y $17 \%$ respectivamente de su recarga natural (Bunge, 2011).

A reserva de conocer la dinámica hídrica de los acuíferos del municipio de Los Cabos, y siguiendo una postura precautoria, Bunge (2011) considera en peligro de intrusión salina si se explota más de $50 \%$ del volumen recargado anualmente, situación que aplica para el acuífero de San José del Cabo.

La dotación de agua podría complicar o comprometer el abasto a las poblaciones locales en el futuro, sobre todo en temporada alta, cuando se observa que el consumo es 183 litros/persona/día. De acuerdo con la Organización Mundial de la Salud (oms), este consumo no es el óptimo para la zona, pues se considera que la cantidad adecuada de agua para consumo humano (beber, cocinar, aseo personal y limpieza del hogar) debe ser de 50 litros/persona/día, aunque en México el promedio asciende a 250 litros/persona/día. Además, el consumo puede aumentar en el futuro, debido a que actualmente los habitantes de esta zona no están conectados a la red de agua potable, lo que los limita, pero no hay garantía de que éste no aumente, sobre todo cuando estén conectados al servicio — se calcula que el consumo podría incrementarse a niveles máximos, como los 600 litros/persona/día que se consumen en viviendas unifamiliares con jardín y piscina en comunidades similares, como las de Valencia, España (Rico-Amorós, 2007). Esta situación, aunada al déficit hídrico y al riesgo de intrusión salina en el acuífero, comprometería la viabilidad de este recurso en el futuro. 


\section{Conclusiones}

El trabajo cumplió su objetivo de profundizar en el conocimiento de los jubilados extranjeros, al caracterizarlos como una comunidad joven de $b a-$ byboomers, provenientes de Estados Unidos y Canadá, de clase media y preparada. Debido a su próxima jubilación, se prevé que el sitio se convierta en un destino de retiro en corto plazo, ya que $49 \%$ de los entrevistados se considera residente y los visitantes pueden pasar temporadas de hasta 10 meses; además, $88 \%$ cuenta con vivienda propia, por lo que consideran seriamente que podría ser su hogar en el retiro a futuro.

Contrario a lo establecido por Scharafran y Monkkone (2011), las implicaciones sobre los servicios públicos no son malas pues las viviendas son autosuficientes; sin embargo, en el caso del agua, puede haber un problema en cuanto a su abastecimiento en el futuro, debido a que el líquido proviene del acuífero de San José, sobreexplotado y en riesgo de intrusión salina; así, su consumo podría elevarse cuando se conecten a la red.

Las implicaciones ambientales de las viviendas son considerables sobre las pérdidas de coberturas, similares o mayores que en zonas de alto desarrollo urbano, con una recuperación muy lenta, sobre todo la VDC, que podría ser la más afectada por el desarrollo y comprometer la seguridad de las viviendas en zona costera. Además, casi toda el área tiene perturbación, con zD que cubren 98\% del ms y $68 \%$ de la VDC, asociado a un patrón de ocupación disperso propiciado por la falta de regulación territorial de las autoridades.

Finalmente, a pesar de las limitaciones de no contar con información de registros oficiales de servicios y del número de viviendas, este trabajo aporta información que permite describir, caracterizar y entender el patrón espacial de la tipología tráiler de Scharafran y Monkkone (2011), en paisajes específicos y reales, evaluar los cambios en sus patrones en el tiempo, y cuantificar los cambios de uso de suelo y pérdida de coberturas resultantes. Asimismo, se enfatiza la necesidad de entender este fenómeno a escala fina en zonas rurales debido al aumento de viviendas fuera de las zonas urbanas. No obstante, es evidente que cuestiones clave como el consumo de agua, el disturbio asociado a las construcciones y la fragmentación del paisaje por caminos, deben abordarse para tener un entendimiento más profundo de este fenómeno. Por otra parte, los resultados de 
este trabajo pueden orientar estudios similares en otras localidades, así como dejar las bases para monitorear la zona en años subsecuentes y, finalmente, proporcionar a las autoridades información básica y clave para llevar a cabo una planificación y regulación más adecuada de este tipo de desarrollo, disminuyendo sus impactos en los ecosistemas y comunidades locales en que se asientan, asegurando su sustentabilidad.

\section{Bibliografía}

Beach, D. (2002), Coastal Sprawl: The Effects of Urban Design on Aquatic Ecosystems in the United States, Pew Oceans Commission, Arlington, Virginia.

Bernier, E. (2003), "El turismo residenciado y sus efectos en los destinos turísticos", Estudios Turísticos, núms. 155-156, pp. 45-70.

Bringas-Rábago, N. L. (1999), "Políticas de desarrollo turístico en dos zonas costeras del Pacífico mexicano", Región y Sociedad, vol. 11, núm. 17, pp. 1-50.

Brooks, M. L. y B. Lair (2005), Ecological Effects of Vehicular Routes in a Desert Ecosystem, US Geological Survey, Recoverability and Vulnerability of Desert Ecosystems Program, Estados Unidos,

Brown, D. G. (2003), "Land Use and Forest Cover on Private Parcels in the Upper Midwest USA, 1970 to 1990", Landscape Ecology, núm. 18, pp. 777-790.

Brunet-Jailly, E. (2005), "Theorizing Borders: An Interdisciplinary Perspective", Geopolitics, vol. 10, núm. 4, pp. 633-649.

Bunge, V. (2011), "Ejercicio preliminar del impacto de la iniciativa de desarrollo turístico Cabo Cortés, B.C.S.", documento de trabajo, Dirección General de Ordenamiento Ecológico y Conservación de Ecosistemas, Instituto Nacional de Ecología, México.

Carrascal, E. y G. Pérez Villegas (1998), "Ocupación territorial y deterioro ambiental ocasionado por la expansión urbano-turística en Acapulco, Guerrero", Investigaciones Geográficas, núm. 37, pp. 111-116.

Casado-Díaz, M. et al. (2004), "Northern European Retired Residents in Nine Southern European Areas: Characteristics, Motivations and Adjustment", Ageing and Society, núm. 24, pp. 353-381.

Coates, K. et al. (2002), "Tracking the Snowbirds: Seasonal Migration from Canada to the USA and Mexico", American Review of Canadian Studies, vol. 32, núm. 3, pp. 433-455.

Cohen, E. (1972), "The Sociology of Tourism: Approaches, Issues, and Findings", Annual Review of Sociology, vol. 10, pp. 373-392. 
Conagua (2005), Aprovechamiento subterráneo de Los Cabos, documento electrónico en hoja de cálculo.

Conway, K. y A. Houtenville (2003), "Out with the Old, in with the Old: A Closer Look at Younger versus Older Elderly Migration”, Social Science Quarterly, núm. 84, pp. 309-328.

Curr, R. F. H. et al. (2000), "Assessing Anthropogenic Impact on Mediterranean Sand Dunes from Aerial Digital Photography", Journal of Coastal Conservation, núm. 6, pp. 15-22.

Dailey, Nancy (2005), When Baby Boom Women Retire, Praeger, Connecticut.

Fisher, R. A. et al. (1922), "The Accuracy of the Planting Method of Estimating the Density of Bacterial Populations", Annals of Applied Biology, núm. 9, pp. 325-359.

Fondo de Población de las Naciones Unidas, UnFPA (2008), "El estado de la población mundial 2007”, en <http://www.unfpa.org.mx/swoP07/unfpaindex.htm>.

Forman, R. T. T. y L. E. Alexander (1998), "Roads and their Major Ecological Effects”, Annual Reviews of Ecology, Evolution, and Systematics, núm. 29, pp. 207231.

Fujita, N. (2004), "Gunnar Myrdal's Theory of Cumulative Causation Revisited", Economic Research, núm. 147.

Gámez, A. y M. Ángeles (2010), “Borders within. Tourism Growth, Migration and Regional Polarization in Baja California Sur, Mexico", Journal of Borderlands Studies, vol. 25, núm. 1, pp. 1-18.

González-Abraham, C. E. et al. (2007), "Building Patterns and Landscape Fragmentation in Northern Wisconsin, USA”, Landscape Ecology, vol. 22, núm. 2, pp. 217-230.

Guaita, N. et al. (2006), Cambios de ocupación del suelo en España: implicaciones para la sostenibilidad, Observatorio de la Sostenibilidad en España, Madrid, España.

Guido, S. (2007), ¿Desarrollo turístico regional? Monitoreo de los desarrollos turísticos e inmobiliarios costeros del noroeste de México, 2005-2006, Alianza para la Sustentabilidad del Noroeste Costero, México.

Hansen, A. J. et al. (2005), "Effects of Exurban Development on Biodiversity: Patterns, Mechanisms, and Research Needs”, Ecological Applications, vol.15, núm. 6, pp. 1893-1905.

Hawbaker, T. J. (2004), "Road Density and Landscape Pattern in Relation to Housing Density, Land Ownership, Land Cover, and Soils”, Landscape Ecology, núm. 20, pp. 609-625.

Hiernaux-Nicolas, D. (2005), "La promoción inmobiliaria y el turismo residencial: el caso mexicano”, Scripta Nova, vol. Ix, núm. 194 (05).

Hoggart, K. (1997), "Rural Migration and Counterurbanization in the European Periphery: The Case of Andalucía”, Sociologia Ruralis, núm. 37, pp. 137-153. 
Hostetler, M. (1999), "Scale, Birds, and Human Decisions: A Potential for Integrative Research in Urban Ecosystems", Landscape and Urban Planning, vol. 45, núm. 1, pp. 15-19.

Huber, A. (2005), "Retirados suizos en la Costa Blanca”, en Vicente Rodríguez (eds.), La migración de europeos retirados en España, Consejo Superior de Investigaciones Científicas, Madrid.

Jasso-Barbosa, M. A. (2007), Estudio comparativo de playas: Mazatlán, Acapulco, Cancún y Los Cabos (1970-2005), III Congreso Internacional de Investigación y Docencia.

Jauhiainen, J. S. (2009), "Will the Retiring Babyboomers Return to Rural Periphery?", Journal of Rural Studies, vol. 25, núm.1, pp. 25-34.

Kiy, R. y A. McEnany (2010), Tendencias sobre la jubilación de estadounidenses en las comunidades costeras de México. Datos demográficos y prioridades en los estilos de vida, International Community Foundation, Estados Unidos.

León de la Luz, J. L. (1999), Flora y vegetación de la Región del Cabo, Baja California Sur, México, tesis de doctorado, Centro de Investigaciones Biológicas del Noroeste.

Lewis, D. et al. (1989), “Counterurbanization in Europe: Discussion”, Geographical Journal, núm.155, pp. 75-80.

Lizárraga-Morales, O. (2008), "Immigration and Transnational Practices of us Retirees in Mexico. A Case Study in Mazatlán, Sinaloa and Cabo San Lucas, Baja California Sur”, Migración y Desarrollo, segundo semestre, pp. 93-110.

— (2009), "Redes e impacto social del turismo residencial de jubilados estadounidenses en Mazatlán, Sinaloa, y Cabo San Lucas, Baja California Sur", Topofilia. Revista de Arquitectura, Urbanismo y Ciencias Sociales, Centro de Estudios de América del Norte, El Colegio de Sonora, vol. I, núm. 3, pp. 1-13. (2010), "The US Citizens Retirement Migration to Los Cabos, Mexico. Profile and Social Effects", Recreation and Society in Africa, Asia Eं Latin America, vol. 1, núm. 1, pp. 75-92.

Mitchell, C. (2004), "Making Sense of Counterurbanization", Journal of Rural Studies, núm. 20, pp. 15-34.

Newman, D. (2006), "Borders and Bordering: Towards an Interdisciplinary Dialogue”, European Journal of Social Theory, vol. 9, núm. 2, pp. 171-186.

Organización Mundial de Turismo (2005), Indicadores de desarrollo sostenible para los destinos turísticos-Guía práctica, Organización Mundial del Turismo, pp. 555.

Pérez-Navarro, J. J. (1995), La vegetación de ambientes costeros de la Región del Cabo, Baja California Sur: aspectos florísticos y ecológicos, tesis de licenciatura, Universidad Nacional Autónoma de México.

Pérez-Villegas, G. y E. Carrascal (2000), "El desarrollo turístico en Cancún, Quintana Roo, y sus consecuencias sobre la cubierta vegetal”, Investigaciones Geográficas, núm. 43, pp. 145-166. 
Raya-Mellado, P. (2001), Turismo residencial en Andalucía, Sevilla, Consejería de Turismo y Deportes de la Junta de Andalucía, España.

Rico Amorós, A. M. (2007), “Tipologías de consumo de agua en abastecimientos urbano-turísticos de la Comunidad Valenciana”, Investigaciones Geográficas, núm. 42, pp. 15-24.

Rosete-Vergés, F. et al. (2008), "Cambio de uso de suelo y vegetación en la península de Baja California, México”. Investigaciones Geográficas, núm. 67, pp. 39-58.

Rzedowski, J. (2006), Vegetación de México. 1a. edición digital, Comisión Nacional para el Conocimiento y Uso de la Biodiversidad Nacional, México.

Saint Onge, J. et al. (2005), Population Growth in High-amenity Rural Areas: Does it Bring Socioeconomic Status Benefits for Long-term Residents? documento de trabajo 7/2005, Institute of Behavioural Science, University of Colorado at Boulder, Estados Unidos.

Schafran, A. y P. Monkkonen (2011), "Beyond Chapala and Cancun: Grappling with the Impact of American Migration to Mexico", Migraciones Internacionales, vol. 6, núm. 2, pp. 223-258.

Seingier, G. et al. (2009), "Cobertura vegetal y marginación en la costa mexicana”, Investigación Ambiental, vol. 1, núm. 1, pp. 54-69.

Smith, V. L. (1977), Hosts and Guests: The Anthropology of Tourism, 2a. edición, University of Pennsylvania Press, Pennsylvania.

Suárez, M. (2004), Interaprendizaje holístico de matemáticas, Planeta, Ecuador.

Theobald, D. M. et al. (1997), "Estimating the Accumulative Effects of Development on Wildlife Habitat”, Landscape and Urban Planning, núm. 39, pp. 25-36.

Vartiainen, P. (1989), "The End of Drastic Depopulation in Rural Finland: Evidence of Counterurbanization?”, Journal of Rural Studies, núm. 5, pp. 123136.

Artículo recibido el 8 de noviembre de 2012. Segunda versión aprobada el 4 de abril de 2013. 